Article

\title{
Condensation: Passenger Not Driver in Atmospheric Thermodynamics
}

\author{
Jack Denur
}

Electric \& Gas Technology, Inc., 3305 Main Street, Rowlett, TX 75088-4983, USA; jackdenur@my.unt.edu; Tel.: +1-214-675-6599

Academic Editors: Eliodoro Chiavazzo and Kevin H. Knuth

Received: 7 April 2016; Accepted: 1 September 2016; Published: 25 November 2016

\begin{abstract}
The second law of thermodynamics states that processes yielding work or at least capable of yielding work are thermodynamically spontaneous, and that those costing work are thermodynamically nonspontaneous. Whether a process yields or costs heat is irrelevant. Condensation of water vapor yields work and hence is thermodynamically spontaneous only in a supersaturated atmosphere; in an unsaturated atmosphere it costs work and hence is thermodynamically nonspontaneous. Far more of Earth's atmosphere is unsaturated than supersaturated; based on this alone evaporation is far more often work-yielding and hence thermodynamically spontaneous than condensation in Earth's atmosphere-despite condensation always yielding heat and evaporation always costing heat. Furthermore, establishment of the unstable or at best metastable condition of supersaturation, and its maintenance in the face of condensation that would wipe it out, is always work-costing and hence thermodynamically nonspontaneous in Earth's atmosphere or anywhere else. The work required to enable supersaturation is most usually provided at the expense of temperature differences that enable cooling to below the dew point. In the case of most interest to us, convective weather systems and storms, it is provided at the expense of vertical temperature gradients exceeding the moist adiabatic. Thus, ultimately, condensation is a work-costing and hence thermodynamically nonspontaneous process even in supersaturated regions of Earth's or any other atmosphere. While heat engines in general can in principle extract all of the work represented by any temperature difference until it is totally neutralized to isothermality, convective weather systems and storms in particular cannot. They can extract only the work represented by partial neutralization of super-moist-adiabatic lapse rates to moist-adiabaticity. Super-moist-adiabatic lapse rates are required to enable convection of saturated air. Condensation cannot occur fast enough to maintain relative humidity in a cloud exactly at saturation, thereby trapping some water vapor in metastable supersaturation. Only then can the water vapor condense. Thus ultimately condensation is a thermodynamically nonspontaneous process forced by super-moist-adiabatic lapse rates. Yet water vapor plays vital roles in atmospheric thermodynamics and kinetics. Convective weather systems and storms in a dry atmosphere (e.g., dust devils) can extract only the work represented by partial neutralization of super-dry-adiabatic lapse rates to dry-adiabaticity. At typical atmospheric temperatures in the tropics, where convective weather systems and storms are most frequent and active, the moist-adiabatic lapse rate is much smaller (thus much closer to isothermality), and hence represents much more extractable work, than the dry-the thermodynamic advantage of water vapor. Moreover, the large heat of condensation (and to a lesser extent fusion) of water facilitates much faster heat transfer from Earth's surface to the tropopause than is possible in a dry atmosphere, thereby facilitating much faster extraction of work, i.e., much greater power, than is possible in a dry atmosphere-the kinetic advantage of water vapor.
\end{abstract}

Keywords: energy; free energy; work; heat; entropy; second law of thermodynamics; kinetics; power; convection; condensation; evaporation 


\section{Introduction: Work, Heat, and Water}

The second law of thermodynamics states that a process is thermodynamically spontaneous only if it yields work or at least is capable of yielding work (irrespective of whether it yields or costs heat): the maximum work that it can possibly yield is its free-energy yield. And that a process that costs work (irrespective of whether it yields or costs heat) is thermodynamically nonspontaneous: the minimum work required to enable it is its free-energy cost [1-11]. These statements are true for all processes, isothermal or not. Two nonisothermal examples: (a) Neutralization of a temperature difference (clearly a nonisothermal process) is capable of yielding work, say, via a heat engine, and hence is thermodynamically spontaneous, but its reverse costs work and hence is thermodynamically nonspontaneous. (b) Adiabatic expansion of a gas (clearly a nonisothermal process) is capable of yielding work, say via a pressure engine, and hence is thermodynamically spontaneous if the external pressure is less than the pressure of the gas, but costs work and hence is thermodynamically nonspontaneous if the external pressure exceeds the pressure of the gas. Of course a thermodynamically spontaneous process can occur without yielding any work and hence with its ability to do work being wasted, e.g., process (a) above without intervention of a heat engine, and process (b) above without intervention of a pressure engine. However, any thermodynamically spontaneous process certainly has the ability to yield work, even though this ability can be wasted. The reverse of any thermodynamically spontaneous process has no ability to do work but costs work, and hence is thermodynamically nonspontaneous [1-11]. (See: Reference [1], Chapter 1; Reference [2], Chapters 12-19, especially Chapters 13, 17, and 19 (in Chapter 17 most especially Section 17.2); Reference [3], especially Chapters I-IV, VII, and VIII; Reference [4], especially Chapters 1-5 and 7; Reference [5], Chapter 8; Reference [6], especially Chapters 1-3, 5, 7, and 10; Reference [7], especially Chapters 1-5; Reference [8], Chapters 2-5, especially Sections 2.1, 2.2, 4.2c, 4.5, and 4.6, and Chapter 5; and Reference [9], Foundations (especially Part B) and Chapters 1-3, especially Part 2A and pp. 131-135. References [8-10] base thermodynamics on the concept of work [11], rather than on the concept of entropy. The advantage of this approach is that work is the most basic, simplest, most sharply defined, and most familiar concept in thermodynamics [8-11], and all more complex thermodynamic concepts, such as energy, heat, entropy, and temperature can be derived from the concept of work [8-11]. Concerning this point, in Reference [8] see most especially Sections $2.2 \mathrm{~b}$ and $2.2 \mathrm{c}$ and the second paragraph of Section 4.2c. Unfortunately, the most valuable of the discussions in Sections 2.1,2.2, and 4.2c were omitted from, or at best stated less completely in, the 8th, 9th, and 10th Editions of Reference [8]. But since the 10th Edition of Reference [8] is the most recent one, we cite it as Reference [9].)

Some clarification concerning the relation between heat and thermodynamic spontaneity may be required: I. A process that yields heat is thermodynamically spontaneous only if it also yields work or at least can yield work. Two examples: (i) A system can spontaneously yield heat to its surroundings only if it is hotter than its surroundings. However, if it is hotter than its surroundings, then a heat engine can employ this temperature difference to yield work. (ii) An exothermic (heat-yielding) chemical or nuclear reaction can occur spontaneously only if it is also exoergic (work-yielding). II. A process that costs heat is thermodynamically spontaneous only if it also yields work or at least can yield work. Two examples: (iii) A system can spontaneously absorb heat from its surroundings only if it is colder than its surroundings. However, if it is colder than its surroundings, then a heat engine can employ this temperature difference to yield work. (iv) An endothermic (heat-costing) chemical or nuclear reaction can occur spontaneously only if it is also exoergic (work-yielding). In example (i) above, the system is in its initial state sensibly hotter than its surroundings; in example (iii) above, sensibly colder. In example (ii) above, the chemical or nuclear reactants can be construed as latently hotter than their surroundings; in example (iv) above, latently colder. Thus for instance the warming of a cold room as per example (i) above via the physical process (sensibly hot object + sensibly cold room $\rightarrow$ sensibly lukewarm object + sensibly lukewarm room) is, in essence, thermodynamically equivalent to warming the room as per example (ii) above via the chemical process of burning coal (latently hot molecules $\left(\mathrm{C}+\mathrm{O}_{2}\right)+$ sensibly cold room $\rightarrow$ latently lukewarm molecules $\left(\mathrm{CO}_{2}\right)+$ sensibly lukewarm 
room). (Note: "exergonic" and "endergonic" are sometimes used as synonyms for "exoergic" and "endoergic", respectively, but mainly pertaining to biochemical reactions.)

A work-costing and hence nonspontaneous process can occur only if enabled by another, spontaneous, process that yields more work than the nonspontaneous one costs [1-11]. It should be emphasized that a work-costing and hence nonspontaneous process would violate the second law of thermodynamics only if it could occur without compensation. A work-costing and hence nonspontaneous process that occurs with compensation, i.e., enabled by another, spontaneous, process that yields more work than the nonspontaneous one costs [1-11], does not violate the second law, because the total process-the sum of both precesses combined-is a work-yielding process.

While the second law has been challenged [12-17], even if some, or even all, of these challenges are borne out it would still have a very wide range of validity: As stated in Reference [14], (p. 13): "If the second law should be shown to be violable, it would nonetheless remain valid for the vast majority of natural and technological processes." The range of validity of the second law would still encompass all processes considered in this paper. Hence for the purposes of this paper we need not consider challenges to the second law [12-17]. (The entireties of References [12-17] concern challenges to the second law of thermodynamics. Hence References [12-17] are cited in their entirety. This includes the Special Issues of Entropy cited in References [13,17] and the Issue of Foundations of Physics cited in Reference [15].)

A thermodynamically neutral process neither yields nor costs work. Thus there is no tendency for such a process to occur, and hence it cannot proceed at a finite rate, in either the forward or reverse direction. An example is a work-costing Process B enabled or driven by an equally work-yielding Process A. The total Process A $+B$ is thermodynamically neutral, and hence cannot proceed at a finite rate. Thus the limit of perfection or reversibility, i.e., Process $B$ recovering all of the work yielded by Process A, is unattainable. In any real such total Process A + B, Process B can recover some-but not all -of the work yielded by Process A, so that the total Process A + B is a work-yielding process. In most-but not all-cases the limit of perfection or reversibility is approached more closely as a process proceeds more slowly. Counterexamples: (a) Adiabatic cooling during cosmological expansion of the Universe or adiabatic heating during its contraction of a sufficiently simple substance such as cosmic background radiation, dust, or possibly a monatomic ideal gas can occur reversibly at a finite rate [18] (In Reference [18] see Sections 130 and 169-171.) (b) Conversely, sliding and rolling friction do not vanish in the limit of vanishing speed, precluding perfection or reversibility from being approached even in this limit [19-23]. (In Reference [19] see Section 6-1.) In fact, sliding friction is greater at vanishing speed than at moderate speed: (coefficient of static sliding friction) $>$ (coefficient of kinetic sliding friction) [19-21]. Hence sliding friction entails greater departure form perfection or reversibility at vanishing speed than at moderate speed [19-21].

The generalized free energy associated with a process is the maximum work this process can yield if it is spontaneous, or the minimum work required to enable it if is nonspontaneous, under any circumstances. The immediately preceding sentence is true with respect to more specialized varieties of free energy (the most commonly employed ones being the Helmholtz and Gibbs free energies) only under restricted circumstances (usually taken to be isothermal, isochoric for the Helmholtz free energy and isothermal, isobaric for the Gibbs free energy). (See: Reference [1], Section 1.27; Reference [2], Chapter 17 (especially pp. 476-479) and Chapter 19; Reference [3], Chapter VIII; Reference [4], Chapters 5 and 7; Reference [5], Sections 8.9-8.13; Reference [6], Chapters 7 and 10, pp. 144-150, Section 11.7, pp. 270-282, and Section 12.10; Reference [7], Chapter 5; Reference [8], pp. 108-134; and Reference [9], pp. 131-135.) However, we primarily consider free energy in the generalized sense indicated in the first sentence of this paragraph. Free energy and its relation to work will be discussed in more detail in Section 3.

We show that in atmospheric thermodynamics condensation is an effect rather than a cause, i.e., a passenger rather than a driver: even though condensation always yields heat, it always costs work. Hence the second law of thermodynamics requires that condensation must be enabled by 
another, work-yielding process that yields more work than condensation costs. Condensation is most usually effected by cooling to below the dew point. In the case of most interest to us, convective weather systems and storms, the work-yielding process entails neutralization of super-moist-adiabatic vertical temperature gradients, hence tending to cool Earth's (land or ocean) surface and warm the tropopause-in accordance with Nature's abhorrence of gradients. The role of water vapor in convective-weather-system thermodynamics is to render available the super-moist-adiabatic portion of vertical temperature gradients and hence of the free energy associated therewith for driving convection. Convection, in turn, enables cooling of ascending air parcels to below the dew point and hence attainment of supersaturation in clouds, because water vapor cannot condense fast enough to maintain relative humidity in clouds exactly at saturation. Supersaturation, in turn, ultimately enables condensation and consequent release of heat of condensation: given supersaturation, condensation of water vapor and consequent release of heat of condensation is thermodynamically spontaneous. However, establishment of supersaturation, and maintenance of supersaturation in the face of condensation that would wipe it out, is itself never thermodynamically spontaneous in Earth's atmosphere or anywhere else. It is driven by convection that is too fast to allow condensation to maintain relative humidity in clouds exactly at saturation, and this convection is in turn driven by super-moist-adiabatic lapse rates. Thus ultimately condensation and consequent release of heat of condensation is a thermodynamicaly nonspontaneous process forced by super-moist-adiabatic lapse rates. The super-moist-adiabatic portion of vertical temperature gradients-not water vapor-is the free energy source.

By contrast, in a dry atmosphere, only the super-dry-adiabatic portion of vertical temperature gradients is available to drive convection. At typical atmospheric temperatures in the tropics, where convective weather systems and storms are most frequent and active, the moist-adiabatic lapse rate is much smaller (thus much closer to isothermality) and hence represents much more extractable work than the dry-the thermodynamic advantage of water vapor. Thus the role of water vapor in atmospheric thermodynamics is to facilitate the extraction of work at the expense of a much larger fraction (the super-moist-adiabatic fraction) of vertical temperature lapse rates and hence of the free energy associated therewith than would be possible without it (the super-dry-adiabatic fraction). Moreover, the large heat of condensation (and to a lesser extent fusion) of water facilitates much faster heat transfer from Earth's surface to the tropopause than is possible in a dry atmosphere, thereby facilitating much faster extraction of work, i.e., much greater power output, than is possible in a dry atmosphere-the kinetic advantage of water vapor. Thus the role of water vapor in convective-weather-system kinetics is to facilitate, via its large heat of condensation (and to a lesser extent fusion), much faster expenditure of this much larger-super-moist-adiabatic as opposed to super-dry adiabatic - fraction of vertical temperature gradients and hence of the free energy associated therewith than would be possible without it. Evaporation at Earth's surface and condensation at high tropospheric altitudes greatly enhances the rate of heat flux from Earth's surface to the tropopause over that attainable in a dry atmosphere.

What is more, the very properties of water help to maintain super-moist-adiabatic lapse rates in Earth's troposphere. Water vapor, because it is a greenhouse gas, raises temperatures at Earth's surface via reradiation of infrared frequencies from Earth's atmosphere (mainly from the troposphere) back to Earth's surface, and enhances radiation of infrared frequencies to space from tropospheric altitudes well above Earth's surface thus lowering temperatures at these higher altitudes. Thus it helps to maintain larger average temperature lapse rates between Earth's surface and the tropopause than would obtain without it. Condensed into clouds, water helps to do the same thing during the nighttime hours: Clouds act as a blanket trapping infrared radiation from Earth's surface and reradiating it back to Earth's surface, thereby raising nighttime temperatures at Earth's surface, while infrared radiation to space from cloud tops, which are better radiators than air, helps to lower nighttime temperatures there. This helps to increase nighttime ambient vertical lapse rates in the air surrounding the clouds. The steepening of lapse rates with the help of water vapor at all times, and with the help of water 
vapor condensed into clouds at night, is greatest where there is the most water vapor-in the humid tropics-which is where convective weather systems and storms are most frequent and active. So it most assists convective weather systems and storms where they need it most. (Of course, during the daytime, clouds usually lower temperatures at Earth's surface: reduced insolation usually more than offsets the heat-blanket effect.)

In Section 2, we discuss condensation and consequent release of heat of condensation, and show that it is always a work-costing and hence nonspontaneous process in Earth's atmosphere. The work-yielding process that pays for it is neutralization of super-moist-adiabatic vertical temperature gradients, i.e., cooling of Earth's (land or ocean) surface and warming of the tropopause-Nature's abhorrence of gradients. Super-moist-adiabatic vertical temperature gradients, indeed all (nongeothermal) temperature disequilibria on Earth, are, in turn, ultimately paid for by the even greater temperature disequilibrium between the $5800 \mathrm{~K}$ solar disk and the $2.7 \mathrm{~K}$ cosmic background radiation [24]. In Section 3, the Helmholtz, Gibbs, and generalized free energies are discussed in some detail. Our preference for generalized free energy is explained. In Section 4 the limitation upon convective weather systems and storms in a saturated atmosphere to extract only the work obtainable by partial neutralization to moist-adiabaticity of vertical temperature gradients with temperature decreasing upwards is discussed. It is contrasted with the more severe limitation upon those in an unsaturated atmosphere to extract only the work obtainable by partial neutralization to dry-adiabaticity of vertical temperature gradients with temperature decreasing upwards, and with the lack of any such limitation on the ability of heat engines in general to extract all of the work obtainable by total neutralization of any temperature gradient irrespective of direction to isothermality. Also in Section 4 convective or hydrodynamic utilizability is contrasted with thermodynamic utilizability. The kinetic acceleration of extraction of work-the increased power-in a saturated atmosphere over that in a dry one is discussed in Section 5. A synopsis is provided in Section 6. Auxiliary topics are discussed in Appendixes A, B, and C.

We do not provide detailed calculations of convective-weather-system thermodynamics. These are provided in many other papers [25-39]. (In Reference [31] (a book) synopses are provided at pp. 12-16 and Chapter 10.) Our main goal is conceptual rather than computational-to show that condensation and consequent release of heat of condensation is always an effect rather than a cause, i.e., always a passenger rather than a driver, in Earth's atmosphere, albeit an important passenger.

\section{Free Energy, Convection, and Condensation}

Condensation is thermodynamically spontaneous and hence can yield work-not merely heat-but only if there is supersaturation, i.e., relative humidity exceeding the saturation value, which is $100 \%$ given a plane surface of pure water. At any given temperature and pressure, the energy of water vapor always exceeds that of liquid water (or of ice), but the Gibbs free energy of water vapor exceeds that of liquid water (or of ice) only given supersaturation [27,30,40] (in Reference [40] see Section 5.6). However, most of Earth's atmosphere is subsaturated. Nevertheless, supersaturation certainly exists in Earth's atmosphere, especially in clouds. The point is that in all such cases the establishment of supersaturation, and its maintenance in the face of condensation that would wipe it out, is itself a work-costing and hence thermodynamically nonspontaneous process, and hence, ultimately, so is condensation and consequent release of heat of condensation that is enabled by supersaturation. Four examples of supersaturation that exist in Earth's atmosphere: (a) Thermal radiation to space from cloud tops, which are better radiators than air, can lower cloud temperatures, especially at and near the tops of clouds, faster than condensation can maintain relative humidity in clouds exactly at saturation, thereby trapping some water vapor in metastable supersaturation. (b) Lapse rates exceeding the moist adiabatic can drive convection and thence condensation faster than condensation can maintain relative humidity in clouds exactly at saturation, thereby trapping some water vapor in metastable supersaturation. (c) At 100\% relative humidity water vapor is at thermodynamic equilibrium with a plane surface of pure water. However, saturation or equilibrium 
relative humidity increases with decreasing water droplet size (owing to a small droplet's curvature water molecules on its surface have fewer neighbors than on a plane surface and hence are less tightly bound and can evaporate more easily) and decreases with increasing nonvolatile solute concentration (solute molecules occupy part of the surface and if hygroscopic also bond with water molecules). Also saturation or equilibrium relative and absolute humidity with respect to metastable supercooled water droplets is higher than with respect to ice crystals at the same (subfreezing) temperature (because water molecules are less tightly bound in the liquid state than in the solid state and hence can evaporate more easily from the former). Thus water can spontaneously evaporate from small and / or low-solute-concentration droplets and condense onto large and/or high-solute-concentration droplets, and similarly spontaneously evaporate from supercooled water droplets and condense onto ice crystals—but never the reverse. (See: Reference [40], Sections 5.8 and 5.10-5.11; Reference [41], Sections 6.1 and 6.5; and Reference [42], Chapter 5.) Note that any one, any two, or all three of these processes (a)-(c) can operate within any given cloud. (d) Supersaturation has been observed in the upper tropical troposphere and lower tropical stratosphere [43]. However: (i) Supersaturation is a metastable state, in all four cases obtaining at the expense of tropospheric vertical super-moist-adiabatic temperature gradients ultimately paid for by insolation from the $5800 \mathrm{~K}$ solar disk heating Earth's surface and the coldness of the $2.7 \mathrm{~K}$ cosmic background radiation allowing radiative cooling of the tropopause [24]. Thus, in all four cases, metastable supersaturation represents temporarily stored free energy derived at the expense of vertical super-moist-adiabatic temperature gradients that are its source. (ii) In all four cases the degree of supersaturation is typically small, well below the maximum possible supersaturation of approximately $400 \%$ relative humidity that obtains in perfectly clean air, at which point water vapor molecules themselves become effective condensation nuclei [44]: even the most pristine tropospheric and even lower stratospheric air almost always contains enough solid condensation nuclei so that condensation can begin at or at least very near the normal dew point [44]. (In Reference [44] see pp. 83-84.) (iii) Concerning Case (d): The upper tropical troposphere and lower tropical stratosphere are so cold that the total amount of water vapor trapped in metastable supersaturation even given its maximum possible value of approximately $400 \%$ relative humidity [44] is bound to be very small. Moreover as noted in (ii) immediately above actual values of supersaturation probably are always well below this maximum [44]. (iv) Thus, in all four cases, supersaturation represents very limited free-energy storage derived at the expense of super-moist-adiabatic vertical temperature gradients that are its source, and ultimately at the expense of the $5800 \mathrm{~K}$-solar-disk/2.7 K-cosmic-background-radiation disequilibrium that continually regenerates these gradients [24]. A vertical temperature lapse rate exceeding the moist adiabatic in a saturated atmosphere between Earth's hot surface and cold tropopause embodies the free energy that yields the work enabling convection and thence supersaturation, condensation, and consequent release of heat of condensation—not vice versa. (See: Reference [7], Section 1.5 (especially Problem 1.40 on p. 27) and Section 5.3 (especially Problem 5.45 on pp. 177-178); Reference [40], Sections 3.3-3.5 and 4.3, pp. 175-177, and Sections 6.5-6.9; Reference [41], Sections 3.4-3.6; Reference [42], Sections 1.2 and 3.5-3.7; and Reference [45], Sections 2.7 and 2.9, and Appendix D.) It embodies free energy rather than merely energy because it can yield work. Once convection is enabled, no additional work is required to condense the water-condensation and consequent release of heat of condensation is a side result of the convective process. However, the work that it costs to enable convection is paid for by the work yielded by neutralization of super-moist-adiabatic temperature gradients, this neutralization tending to cool Earth's (land or ocean) surface and warm the tropopause-by Nature's abhorrence of gradients. Only if convection is thus enabled can the side result of supersaturation and thence condensation and consequent release of heat of condensation then also be enabled. It costs work-the work that it costs to enable convection - to force supersaturation and thence condensation and consequent yielding of heat of condensation: supersaturation and thence condensation and consequent yielding of heat of condensation is thus a thermodynamically nonspontaneous process that must be forced. 
Thus in all cases of supersaturation in convective weather systems and storms, the free-energy hierarchy is: $5800 \mathrm{~K}$-solar-disk/2.7 K-cosmic-background-radiation disequilibrium $\rightarrow$ tropospheric vertical super-moist-adiabatic temperature gradients $\rightarrow$ convection $\rightarrow$ cooling of ascending air parcels to below the dew point $\rightarrow$ supersaturation $\rightarrow$ condensation and consequent release of heat of condensation [24]. Condensation is a work-yielding and hence thermodynamically spontaneous given supersaturation. However, establishment of supersaturation, and its maintenance in the face of condensation that would wipe it out, is itself a work-costing and hence thermodynamically nonspontaneous process that can occur only because tropospheric vertical super-moist-adiabatic temperature gradients can yield more work than establishment and maintenance of supersaturation costs. Thus ultimately condensation and consequent release of heat of condensation can occur only because tropospheric vertical super-moist-adiabatic temperature gradients can yield more work than establishment and maintenance of supersaturation costs.

Perhaps this point is most clearly made evident by considering the optimum circumstance for generation and maintenance of convective weather systems and storms with a sub-moist-adiabatic vertical temperature gradient. This optimum circumstance with a sub-moist-adiabatic lapse rate obtains if the entire troposphere is saturated at $100 \%$ relative humidity and however high an absolute humidity, with however high an equivalent potential temperature $\theta_{e}$ (the temperature of air if brought moist-adiabatically to a pressure of 1000 mbar). Release of heat of condensation limits the rate of cooling of saturated ascending air parcels to the moist adiabatic, resulting in positive buoyancy and thence in further convection - but only if the environmental lapse rate exceeds the moist adiabatic. Even given the optimum circumstance with a sub-moist-adiabatic environmental lapse rate-the entire troposphere saturated at $100 \%$ relative humidity and however high an absolute humidity, with however high an equivalent potential temperature $\theta_{e}$-buoyancy is negative even with the heat of condensation. Thus even given this optimum circumstance with a sub-moist-adiabatic lapse rate, the atmosphere is incapable of doing the work necessary to enable convection, thence supersaturation as per Item (b) of the first paragraph of this Section 2, and finally the goal of condensation and consequent release of heat of condensation - despite the heat of condensation being equally large at any given temperature whether the lapse rate is sub- or super-moist-adiabatic. Thus even given this optimum circumstance with a sub-moist-adiabatic lapse rate, a jump from saturation to supersaturation-let alone a jump from subsaturation to supersaturation - cannot be made, and hence condensation and consequent release of heat of condensation cannot occur. Thus there can then be no convection, hence no supersaturation, and thence no condensation and no release of heat of condensation. Convective weather systems and storms-not even the smallest fair-weather cumulus clouds, let alone thunderstorms, tornadoes, and tropical cyclones-could not then exist. (See: Reference [40], Chapters 5-6; Reference [41], Sections 8.3 and 8.4; and Reference [45], Sections 9.5-9.7.)

Thus supersaturation and thence condensation and consequent release of heat of condensation can occur only via being enabled, albeit as a side result of convection being enabled, by a process that yields work. Neutralization of any temperature gradient is capable of yielding work. In the case currently under consideration, neutralization of a super-moist-adiabatic vertical temperature gradient-albeit only partial neutralization to the moist adiabatic and not total neutralization to isothermality because the sub-moist-adiabatic portion is convectively or hydrodynamically unutilizable-yields the work required to enable convection, thence supersaturation, and ultimately condensation and consequent release of heat of condensation. (Convective or hydrodynamic utilizability will be discussed more thoroughly, and contrasted with thermodynamic utilizability, in Section 4.) Owing to release of heat of condensation, heat transfer from Earth's surface to the tropopause is more effective than would be possible without it, thereby facilitating this partial neutralization—but this partial neutralization drives condensation and consequent release of heat of condensation, and not vice versa.

The main point is that a super-moist-adiabatic lapse rate enables condensation and consequent release of heat of condensation, but condensation and consequent release of heat of condensation does not enable a super-moist-adiabatic lapse rate. A super-moist-adiabatic lapse rate is the cause and 
condensation is the effect, and not vice versa. This is what we mean by a super-moist-adiabatic lapse rate being the driver and condensation being the passenger, albeit an important passenger.

Of course it is well known that Earth's atmosphere is always out of equilibrium, and that it is maintained out of equilibrium only at the expense of the even greater disequilibrium between the $5800 \mathrm{~K}$ solar disk and the $2.7 \mathrm{~K}$ cosmic background radiation [24]. The disequilibrium between the $5800 \mathrm{~K}$ solar disk and the $2.7 \mathrm{~K}$ cosmic background radiation [24] is at the top of the free-energy hierarchy $5800 \mathrm{~K}$-solar-disk $/ 2.7 \mathrm{~K}$-cosmic-background-radiation disequilibrium $\rightarrow$ tropospheric vertical super-moist-adiabatic temperature gradients $\rightarrow$ convection $\rightarrow$ cooling of ascending air parcels to below the dew point $\rightarrow$ supersaturation $\rightarrow$ condensation and consequent release of heat of condensation [24]. Hence the disequilibrium between the $5800 \mathrm{~K}$ solar disk and the $2.7 \mathrm{~K}$ cosmic background radiation [24] is the ultimate driver of all organized (nongeothermally-driven and nontidal) activity-meteorological or otherwise-on Earth. But it is nonetheless of interest to inquire concerning, and to distinguish between, immediate drivers (immediate causes) and passengers (effects).

Wind drives (causes) swaying of tree branches and not vice versa. Thus wind is the immediate driver (immediate cause) and swaying of tree branches is the passenger (effect), and not vice versa. Likewise, super-moist-adiabatic lapse rate drives (causes) condensation and consequent release of heat of condensation in convective weather systems and storms, and not vice versa. Thus super-moist-adiabatic lapse rate is the immediate driver (immediate cause), and condensation and consequent release of heat of condensation is the passenger (effect), and not vice versa. The "tree theory" of wind, according to which wind is driven (caused) by trees swaying their branches, is wrong. Likewise, the "condensation theory" of convective weather systems and storms, according to which convective weather systems and storms are driven (caused) by condensation and consequent release of heat of condensation, is wrong. Both theories are wrong for the same reason: Setting of tree branches to swaying and condensation of water vapor are both work-costing and hence thermodynamically nonspontaneous processes in Earth's atmosphere. A work-costing process can occur only if driven by a work-yielding process that yields more work than the work-costing one costs. Tree branches sway only because braking of wind yields more work than it costs to set them swaying. Condensation and consequent release of heat of condensation occurs in convective weather systems and storms only because neutralization of super-moist-adiabatic lapse rate towards the moist adiabatic yields more work than condensation and consequent release of heat of condensation costs.

Vertical temperature gradient exceeding the moist adiabatic is the major free-energy source that yields the work enabling convection and thence supersaturation, condensation, consequent release of heat of condensation, and convective weather systems and storms. While other, auxiliary, free-energy sources may yield some contribution, they cannot generate or maintain convective weather systems and storms in the face of a sub-moist-adiabatic lapse rate. For example, kinetic (wind) energy imported from the surrounding atmosphere is in many cases a significant auxiliary free-energy source for convective weather systems and storms, but still only an auxiliary one: it cannot generate or maintain convective weather systems and storms in the face of a sub-moist-adiabatic lapse rate.

\section{Helmholtz, Gibbs, and Generalized Free Energy}

The type of free energy that is expended by convective weather systems and storms should be specified. The two most commonly employed types are the Helmholtz and Gibbs free energies. Employing the standard notations $P$ for pressure, $V$ for volume, $E$ for internal energy, $S$ for entropy, and $H \equiv E+P V$ for enthalpy, the differential changes in the Helmholtz function $A \equiv E-T S$ and 
Gibbs function $G \equiv H-T S \equiv E+P V-T S$ (not yet specified as the Helmholtz and Gibbs free energies) of a system are, respectively,

$$
\begin{aligned}
d A & \equiv d E-d(T S)=d E-T d S-S d T \\
& =d Q_{\text {in }}-d W_{\text {out }}-T d S-S d T \\
& =-d W_{\text {out }}-S d T \\
& =-p d V-S d T \text { ( } p d V \text { work only) }
\end{aligned}
$$

and

$$
\begin{aligned}
d G & \equiv d H-d(T S)=d H-T d S-S d T \\
& =d E+d(P V)-d(T S)=d E+P d V+V d P-T d S-S d T \\
& =d Q_{\text {in }}-d W_{\text {out }}+P d V+V d P-T d S-S d T \\
& =-d W_{\text {out }}+P d V+V d P-S d T \\
& =-P d V+P d V+V d P-S d T(p d V \text { work only) } \\
& =V d P-S d T .
\end{aligned}
$$

In the second line of Equation (1) we apply the first law of thermodynamics in the form $d E=d Q_{\text {in }}-d W_{\text {out }}$. Taking the limit of reversibility, in the third and fourth lines of Equation (1) $d Q_{\text {in }}=T d S$ and $d W_{\text {out }}=P d V$, respectively. In the second line of Equation (2) we apply $H \equiv E+P V$, and in the third line thereof the first law of thermodynamics in the form $d E=d Q_{\mathrm{in}}-d W_{\text {out }}$. Taking the limit of reversibility, in the fourth and fifth lines of Equation (2) $d Q_{\text {in }}=T d S$ and $d W_{\text {out }}=P d V$, respectively. Note that, strictly, in Equations (1) and (2) $P$ is the external pressure acting on the system of interest, but since we are considering the limit of reversibility $P$ is also the internal pressure of the system of interest. Since pressure, volume, temperature, and entropy are all state functions, the Helmholtz function $A$ and the Gibbs function $G$ are also state functions. (Note that the last lines of Equations (1) and (2), if taken per mole, are the Gibbs-Duhem relations for a single-component system pertinent to the Helmholtz and Gibbs free energies, respectively. For simplicity we consider single-component systems with fixed numbers of particles, i.e., with fixed $N$. The single-component water-only system is of most interest to us (other components of air mixed with water vapor have negligible effects: see Reference 40, Section 5.7. If a single-component system can exchange like particles with its surroundings, then the term $d(\mu N)=\mu d N+N d \mu$ must be added to Equations (1) and (2), where $\mu=(\partial E / \partial N)_{S, V}=(\partial H / \partial N)_{S, P}=(\partial A / \partial N)_{T, V}=(\partial G / \partial N)_{T, P}$ is the chemical potential). For multi-component systems a $d(\mu N)=\mu d N+N d \mu$ term for each component must be added to Equations (1) and (2).)

We are interested in special cases wherein the Helmholtz function $A$ and the Gibbs function $G$ are not merely state functions but $u s e f u l$ state functions, i.e., state functions with a useful physical interpretation. Henceforth we will consider only special cases wherein $A$ and $G$ are useful state functions: the Helmholtz free energy and Gibbs free energy, respectively. It is usually asserted that the Helmholtz function $A$ is a useful state function only in the special case of isothermal, isochoric conditions and that the Gibbs function $G$ is a useful state function only in the special case of isothermal, isobaric conditions. For in the special case of isothermality and isochoricity, the Helmholtz function $A$ is the Helmholtz free energy: In this special case the magnitude $|\Delta A|$ of the Helmholtz-free-energy change $\Delta A$ associated with a process is the maximum work this process can yield if it is spontaneous, or the minimum work required to enable it if it is nonspontaneous. Likewise, in the special case of isothermality and isobaricity, the Gibbs function $G$ is the Gibbs free energy: In this special case the magnitude $|\Delta G|$ of the Gibbs-free-energy change $\Delta G$ associated with a process is the maximum work this process can yield if it is spontaneous, or the minimum work required to enable it if it is nonspontaneous.

To be precise, the restrictions with respect to temperature can be slightly relaxed, with $A$ and $G$ still being the useful state functions described in the immediately preceding paragraph (see Reference [2], pp. 476-479). For our special-case definition of $\Delta A$ as the Helmholtz-free-energy change of a system 
to be valid, the volume of a system undergoing a process must be maintained strictly constant (i.e., the process must be strictly isochoric), but the temperature of the system can vary in intermediate states of a process so long as at the very least it is at the same temperature $T$ in the initial and final states. Likewise, for our special-case definition of $\Delta G$ as the Gibbs-free-energy change of a system to be valid, a system undergoing a process must be in contact with a reservoir that maintains a strictly constant external pressure (i.e., the process must be strictly isobaric), but the temperature of the system can vary in intermediate states of a process so long as at the very least it is at the same temperature $T$ in the initial and final states.

Indeed, another relaxation of restrictions, with $A$ and $G$ still being these useful state functions, seems possible. For, surely, since $T$ and $V$ are the natural independent variables for $A$, by Equation (1) under isothermal conditions in the limit of reversibility $d A=-p d V=-d W_{\text {out,max }}=d W_{\mathrm{in}, \min }$. Likewise, equally surely, since $T$ and $P$ are the natural independent variables for $G$, by Equation (2) under isothermal conditions in the limit of reversibility $d G=V d P=-d W_{\text {out,max }}=d W_{\text {in,min. }}$. (In the fifth line of Equation (2) we equate $-d W_{\text {out }}$ with $-P d V$ rather than with $V d P$ so that in the last line of Equation (2) $d G$ is expressed in terms of its natural independent variables $d T$ and $d P$.) Thus, under isothermal conditions, the magnitude $|\Delta A|=\left|\int p d V\right|$ is the maximum work $W_{\text {out,max }}$ that an increase in the volume of a system can yield, or the minimum work $W_{\text {in,min }}$ required to enable a decrease of its volume. Likewise, under isothermal conditions, the magnitude $|\Delta G|=\left|\int V d p\right|$ is the maximum work $W_{\text {out,max }}$ that can be yielded via a system being allowed to experience a decrease in pressure, or the minimum work $W_{\text {in,min }}$ required to impose an increase in pressure upon it.

Yet, even with both of these relaxations of restrictions, the Helmholtz and Gibbs free energies $A$ and $G$ do not seem to be the most general useful state functions, physically interpretable in term of work. In atmospheric processes, temperature, pressure, and volume occupied per unit mass are typically not constant, with even initial and final states typically of unequal temperature, unequal pressure, and unequal volume occupied per unit mass. Hence neither the Gibbs free energy $G$ nor the Helmholtz free energy $A$ seem to always be the best, or at least the most general, choice for atmospheric processes. Nonetheless it should be noted that the Gibbs free energy has been employed in detailed calculations of convective-weather-system thermodynamics [27,30]. Also, the vapor pressure at which water vapor is at thermodynamic equilibrium with liquid water (or ice) at any given temperature, with no net tendency towards either condensation or evaporation, is that corresponding to the Gibbs free energy of water vapor being equal to that of liquid water (or ice) $[27,30,40]$. Of course, this is true with respect to not only water, but all substances.

However, both the Helmholtz and Gibbs free energies are special cases of the generalized free energy $F$. The magnitude $|\Delta F|$ of the generalized-free-energy change $\Delta F$ associated with a process is the maximum work this process can yield if it is spontaneous, or the minimum work required to enable it if it is nonspontaneous, under whatever conditions of temperature, pressure, and volume occupied per unit mass. The differential change $d F$ in the generalized free energy corresponding to a given differential process is

$$
\begin{aligned}
d F & =-T_{\text {cold }} d S_{\text {total }}=-T_{\text {cold }} d\left(S_{\text {system }}+S_{\text {surrouundings }}-S_{\text {interaction }}\right) \\
& \doteq-T_{\text {cold }} d\left(S_{\text {system }}+S_{\text {surrouundings }}\right)
\end{aligned}
$$

where $d S_{\text {total }}$ is the differential change in total entropy $S_{\text {total }}=S_{\text {system }}+S_{\text {surrouundings }}-S_{\text {interaction }}$ of the system of interest plus its surroundings combined including their interaction corresponding to this given differential process, and $T_{\text {cold }}$ is the temperature of the coldest available heat sink (the tropopause for convective weather systems and storms). The surroundings could be the heat sink itself but in any case must at least incorporate the heat sink. Interaction correlates a system with its surroundings and thus contributes negative entropy (negentropy) $-S_{\text {interaction }}$ to $S_{\text {total }}$. However, in all cases of interest to us, $\left|S_{\text {interaction }}\right|$ is negligible and hence so is the consequent nonadditivity of entropy and of changes 
in entropy with respect to $S_{\text {system }}$ and $S_{\text {surrouundings }}$ exclusively, as per the second line of Equation (3). (The dot-equal sign $(\doteq)$ means "very nearly equal to".)

Note that: (a) The concept of generalized free energy $F$ is equivalent to the concept of available work if the heat sink is construed as being included within the boundary of our system, so that by the first law of thermodynamics $d E=0$ (see Reference [2], Section 17.2). (b) The location of the boundary of a system is arbitrary, and hence has no effect on thermodynamic behavior. For example, it is thermodynamically equivalent to construe that System A does $P d V$ work on an external System B, or to construe that this $P d V$ work is done internally within the isolated combined System $\mathrm{A}+\mathrm{B}$. (c) Our definition of $\Delta F$ remains valid even if $T_{\text {hot }}$ and/or $T_{\text {cold }}$ not only vary in intermediate states, but are not the same even in the initial and final states (The incorporation of $T_{\text {hot }}$ into $\Delta F$ (or $d F$ ) and hence into Equation (3) will be explained in the paragraph containing Equations (6) and (7).) (d) In general entropy and temperature are state functions, and thus $S_{\text {system }}, S_{\text {surrouundings }}, S_{\text {interaction }}, T_{\text {cold }}$, and $T_{\text {hot }}$ in particular are state functions ( $\left|S_{\text {interaction }}\right|$ being negligible in all cases of interest to us). Hence the generalized free energy $F$ is a state function. (e) Unlike $A$ and $G, F$ is always a useful state function, i.e., always a free energy: the magnitude $|\Delta F|$ of the generalized-free-energy change $\Delta F$ associated with a process always is the maximum work this process can yield if it is spontaneous, or the minimum work required to enable it if it is nonspontaneous, under whatever conditions of temperature, pressure, and volume occupied per unit mass. Moreover, as we will now show, both the Helmholtz and Gibbs free energies are special cases of the generalized free energy.

The differential change in the Helmholtz free energy is

$$
\begin{aligned}
d A & =d E_{\text {system }}-T d S_{\text {system }}=-T d S_{\text {surrouundings }}-T d S_{\text {system }} \\
& =-T\left(d S_{\text {system }}+d S_{\text {surrouundings }}\right)=-T d S_{\text {total }} \\
& =\text { special case of } d F
\end{aligned}
$$

The first step in the first line of Equation (4) implicitly, and the second step therein more explicitly, entails the assumption that $\left|S_{\text {interaction }}\right|$ and hence the consequent nonadditivity of entropy and of changes in entropy with respect to $S_{\text {system }}$ and $S_{\text {surrouundings }}$ exclusively is negligible, as per the second line of Equation (3). The second step in the first line of Equation (4) is justified because, given the validity of the definition of $\Delta A$ in terms of work if an entire process is isochoric, i.e., with volume fixed and if the system of interest is at the same temperature $T$ at the very least in the initial and final states, $d E_{\text {system }}=-T d S_{\text {surrouundings }}$. Similarly, the differential change in the Gibbs free energy is

$$
\begin{aligned}
d G & =d H_{\text {system }}-T d S_{\text {system }}=-T d S_{\text {surrouundings }}-T d S_{\text {system }} \\
& =-T\left(d S_{\text {system }}+d S_{\text {surrouundings }}\right)=-T d S_{\text {total }} \\
& =\text { special case of } d F
\end{aligned}
$$

The first step in the first line of Equation (5) implicitly, and the second step therein more explicitly, entails the assumption that $\left|S_{\text {interaction }}\right|$ and hence the consequent nonadditivity of entropy and of changes in entropy with respect to $S_{\text {system }}$ and $S_{\text {surrouundings }}$ exclusively is negligible, as per the second line of Equation (3). The second step in the first line of Equation (5) is justified because, given the validity of the definition of $\Delta G$ in terms of work if an entire process is isobaric, i.e., with ambient pressure fixed, and if the system of interest is at the same temperature $T$ at the very least in the initial and final states, $d H_{\text {system }}=-T d S_{\text {surrouundings. }}$. Thus Equation (4) is a special isochoric case of Equation (3), and Equation (5) is a special isobaric case of Equation (3), in both special cases with the system at the same temperature $T$ at the very least in the initial and final states. Hence both the Helmholtz and Gibbs free energies are special cases of the generalized free energy.

The differential form as employed in Equation (3) is most applicable for our purposes, and is also employed in Equations (4) and (5) so that as special cases they are expressed in the same form as Equation (3). In convective meteorological systems such as thunderstorms, tornadoes, and tropical 
cyclones, heat transfer from the heat source at Earth's (land or ocean) surface at temperature $T_{\text {hot }}$ to the heat sink at the tropopause at temperature $T_{\text {cold }}$ may be sufficiently large and rapid that the decrease in $T_{\text {hot }}$ of Earth's surface and/or the increase in $T_{\text {cold }}$ of the tropopause during the progress of a storm cannot be neglected: hence the differential form as employed in Equation (3) is the most applicable form. Of course, any differential equation can be integrated if we wish to consider an entire process or any finite portion thereof. Note again that our definition of $\Delta F($ or $d F)$ in terms of work remains valid even if $T_{\text {hot }}$ and/or $T_{\text {cold }}$ not only vary in intermediate states, but are not the same even in the initial and final states.

In accordance with the first and second laws of thermodynamics, $T_{\text {hot }}$ is incorporated into $\Delta F$ (or $d F$ ) and hence into Equation (3) via $d S_{\text {total }}$. Corresponding to a given differential heat increment $d Q_{\text {in,hot }}$ extracted from Earth's (land or ocean) surface, a given differential heat increment $d Q_{\text {out,cold }}$ deposited at the tropopause, a given differential increment $d W_{\text {out,actual }}$ of work actually done, and the maximum theoretical differential increment $d W_{\text {out max }}$ of work allowed by the second law of thermodynamics corresponding to $d S_{\text {total }}=0$ in general and hence to Carnot efficiency $\epsilon_{\text {Carnot }}$ for heat engines operating in a cycle in particular, we have [46] (in Reference [46] see Sections 5.11 and 5.12 and Problem 5.22 on p. 199):

$$
\begin{aligned}
d S_{\text {total }} & =\frac{d Q_{\text {out,cold }}}{T_{\text {cold }}}-\frac{d Q_{\text {in,hot }}}{T_{\text {hot }}}=\frac{d Q_{\text {in,hot }}-d W_{\text {out,actual }}}{T_{\text {cold }}}-\frac{d Q_{\text {in,hot }}}{T_{\text {hot }}} \\
& =\left(\frac{1}{T_{\text {cold }}}-\frac{1}{T_{\text {hot }}}\right) d Q_{\text {in,hot }}-\frac{d W_{\text {out,actual }}}{T_{\text {cold }}}=\frac{T_{\text {hot }}-T_{\text {cold }}}{T_{\text {cold }} T_{\text {hot }}} d Q_{\text {in,hot }}-\frac{d W_{\text {out,actual }}}{T_{\text {cold }}} \\
& =\frac{\frac{T_{\text {hot }}-T_{\text {cold }}}{T_{\text {hot }}} d Q_{\text {in,hot }}-d W_{\text {out,actual }}}{T_{\text {cold }}}=\frac{\left(1-\frac{T_{\text {cold }}}{T_{\text {hot }}}\right) d Q_{\text {in,hot }}-d W_{\text {out,actual }}}{T_{\text {cold }}} \\
& =\frac{\epsilon_{\text {Carnot }} d Q_{\text {in,hot }}-d W_{\text {out,actual }}}{T_{\text {cold }}}=\frac{d W_{\text {out max }}-d W_{\text {out,actual }}}{T_{\text {cold }}} \geq 0 \\
& \Longrightarrow d F=-T_{\text {cold }} d S_{\text {total }}=d W_{\text {out,actual }}-d W_{\text {out,max }}=d W_{\text {out,actual }}-d F_{\text {source }} \leq 0 .
\end{aligned}
$$

The first law of thermodynamics, in the form $d Q_{\text {out,cold }}=d Q_{\text {in,hot }}-d W_{\text {out,actual }}$, is applied in the second step of the first line of Equation (6). The second law of thermodynamics requires $d S_{\text {total }} \geq 0$ and hence $d W_{\text {out,actual }} \leq d W_{\text {outmax }}$ or equivalently $d W_{\text {out,actual }} \leq d F_{\text {source, }}$ where $d F_{\text {source }}$ is the differential loss of free energy by the source thereof considered alone, not to be confused with the net differential change in free energy $d F: d F_{\text {source }}$ is lost by a super-moist-adiabatic gradient upon its differential neutralization, this loss being partially recovered as $d W_{\text {out,actual }}<d W_{\text {out,max }}$ performed by a convective weather system or storm. The equalities of the $\geq$ and $\leq$ signs in the last two lines of Equation (6) obtain only in the limit of perfection or reversibility; only in this (unattainable) limit would $d F_{\text {source }}$ lost by the gradient be fully recovered as $d W_{\text {out,actual }}=d W_{\text {out,max }}$ performed by a convective weather system or storm. The truest thermodynamic measure of efficiency is not the Carnot efficiency $\epsilon_{\text {Carnot }}$ but the work efficiency

$$
\begin{aligned}
\epsilon_{\text {work }} & =\frac{d W_{\text {out,actual }}}{d W_{\text {out,max }}}=\frac{d W_{\text {out,actual }}}{d F_{\text {source }}} \text { in general } \\
& =\frac{\epsilon}{\epsilon_{\text {Carnot }}}=\frac{\epsilon}{1-\frac{T_{\text {cold }}}{T_{\text {hot }}}} \text { for heat engines operating in a cycle. }
\end{aligned}
$$

If as in tropical cyclones frictional dissipation of $W_{\text {out,actual }}\left(d W_{\text {out,max }}\right.$ in the (unattainable) limit of perfection or reversibility) is into the hot reservoir at Earth's (typically ocean [31-39] but untypically desert [38] (see also Reference [39], Endnote 10) surface rather than into the cold reservoir at the tropopause, then the cost required of the hot reservoir is reduced from $d Q_{\text {in,hot }}$ to $d Q_{\text {in,hot }}-d W_{\text {out,actual }}$ (to $d Q_{\text {in,hot }}-d W_{\text {out,max }}$ in the (unattainable) limit of perfection or reversibility). Tropical-cyclone performance is thereby enhanced. Dissipation into the hot reservoir also has been employed to enhance the performance of thermoelectric generators [47]. 
The contribution to hurricane intensity by frictional dissipation and thence recycling of a hurricane's work output (wind) into its hot reservoir has been investigated for typical hurricanes, whose hot reservoir is the tropical ocean surface. Such recycling must also contribute, even if only slightly, to (re)intensification over land, rather than over water, as tropical or warm-core (not extratropical or cold-core) cyclones, of untypical hurricanes in the tropical deserts of northern Australia, whose hot reservoir is the sandy desert soil [38] (see also Reference [39], Endnote 10). The major cause is the increased thermal conductivity of the sandy desert soil when moistened by the hurricane's own rainfall, which more than compensates for the negative effects of cooling of the soil by this same rainfall. Recycling of a hurricane's work output (wind) via friction into this hot reservoir must contribute, even if only slightly, towards partially counteracting this rain-cooling.

Contrasting energy sources versus free-energy sources: A free-energy source is a source solely of disequilibrium, or equivalently of localization or negentropy — not at all of energy. Note well that by Equations (3)-(6) a differential change of free energy is proportional solely to a differential change of total entropy $d S_{\text {total }}$ (or total negentropy $-d S_{\text {total }}$ )—not at all to a differential change of total energy. Indeed by the first law of thermodynamics even a differential or infinitessimal change of total energy is impossible. Energy is incorporated into free energy only via multiplication of $d S_{\text {total }}$ by $T_{\text {cold }}$. The dimensions of free energy are indeed those of energy, not those of entropy or negentropy, but these dimensions obtain only via multiplication of $d S_{\text {total }}$ by $T_{\text {cold }}-$ it is $d S_{\text {total }}$ and not $T_{\text {cold }}$ that provides the drive (except via contributing to $d S_{\text {total }}$ by being lower than $T_{\text {hot }}$ ). A free-energy source can be the disequilibrium between ambient and either an energy source or an energy sink-burning fuel can run a heat engine but so can liquid nitrogen, ice, or evaporating water [48-58] (Summaries of Reference [52] are provided in References [53,54], and summaries of Reference [56] are provided in References [57,58]. An auxiliary website is available online for Reference [58].) Disequilibrium is localization: a heat engine running on burning fuel expends localization of energy (not energy itself) from the heat source (burning fuel), delocalizing this energy into the ambient environment; one running on liquid nitrogen, ice, or evaporating water [48-58] expends localization of energy (not energy itself) in the ambient environment, delocalizing this energy into the heat sink (liquid nitrogen, ice, or evaporating water). Evaporation of water into an unsaturated atmosphere (relative humidity less than 100\%) is a thermodynamically spontaneous process, yielding work even though it costs heat [48-58]. Since most of Earth's atmosphere is unsaturated, in most of Earth's atmosphere evaporation—not condensation-is the work-yielding and hence thermodynamically spontaneous process, even though evaporation always costs heat and condensation always yields heat. This is even neglecting that establishment of supersaturation, and its maintenance in the face of condensation that would wipe it out, is always work-costing and hence thermodynamically nonspontaneous in Earth's atmosphere or anywhere else, and hence that even in supersaturated regions of Earth's or any other atmosphere condensation is ultimately always work-costing and hence thermodynamically nonspontaneous. The work yielded by evaporation of water sets into motion heat engines including but not limited to drinking birds and toy cars [48-58]. It also sets thunderstorm downburst winds into motion (with the help of the weight of raindrops, and sometimes also with the help of melting of hail, which, like evaporation into an unsaturated atmosphere (relative humidity less than 100\%), yields work even though it costs heat). (See: Reference [41], pp. 350-352 and 361-362; and Reference [42], Sections 5.7, 8.2, and 8.10, and pp. 268-270.) It also helps to lift water up trees [48]. Research and development towards practical evaporation-powered engines for numerous applications has been undertaken [52-54] and is currently underway [55-58]. Thus there are evaporation-driven engines [48-58] but not condensation-driven engines: Convective weather systems and storms are lapse-rate-driven engines, not condensation-driven engines. Evaporation yields work in accordance with Equations (3) and (6): Evaporation costs heat and thus costs localization in momentum space and hence a decrease in momentum-space or thermal entropy-the width at half maximum of the Maxwellian velocity, or, better, momentum distribution decreases with decreasing temperature. However, in an unsaturated atmosphere (relative humidity less than 100\%) delocalization of water molecules in position space increases enough upon evaporation to yield a more-than-compensating 
increase in position-space or configurational entropy. This delocalization in position space upon evaporation occurs, first, owing to breaking of correlations between water molecules, and second, after these correlations have been broken, owing to expansion in position space of the water vapor. Thus upon evaporation into an unsaturated atmosphere there occurs net delocalization in the total momentum-plus-position phase space, and hence the total thermal-plus-configurational entropy $S_{\text {total }}$ increases. (As already noted in the second sentence of Section 2, at any given temperature and pressure, the energy of water vapor always exceeds that of liquid water (or ice), but the Gibbs free energy of water vapor exceeds that of liquid water (or ice) only given supersaturation $[27,30,40]$.) To summarize this paragraph: In all cases the total energy of a system plus its surroundings combined is constant: Work is always obtained solely at the expense of delocalization of energy, i.e., at the expense of expenditure of negentropy, never at the expense of expenditure of energy. (One point: On p. 479 of Reference [2] it is stated that in an adiabatic process all of the energy lost by a system can be converted to work, but that in a nonadiabatic process less than all of the energy lost by a system can be converted to work. However, if the entropy of a system undergoing a nonadiabatic process increases, then more than all of the energy lost by this system can be converted to work, because energy extracted from the surroundings can then also contribute to the work output. In some such cases positive work output can be obtained at the expense of the surroundings even if the change in the system's energy is zero, indeed even if the system gains energy. Two examples: (a) Isothermal expansion of an ideal gas yields work even though the energy change of the ideal gas is zero. (b) As per this paragraph and References [48-58], evaporation of liquid water (or ice) into an unsaturated atmosphere (relative humidity less than $100 \%$ ) yields work even though liquid water (or ice) gains energy in becoming water vapor. Both examples (a) and (b) immediately above are thermodynamically spontaneous processes; their reverses are nonspontaneous. They both entail delocalization of energy from the surroundings into the system. This emphasizes that delocalization of energy rather than energy per se enables work.)

\section{Limitations on Convectively Utilizable Free Energy: Thermodynamic Versus Hydrodynamic Utilizability}

The second law of thermodynamics allows, at least in principle, heat engines in general to extract all of the work represented by any temperature gradient via its convective, conductive, and/or radiative neutralization, until the gradient is totally neutralized to isothermality. Also the spatial direction of the temperature gradient is immaterial: heat engines in general can extract all of the work represented by an inversion with temperature decreasing downwards equally as well that represented a "normal" gradient with temperature decreasing upwards, until either gradient is totally neutralized to isothermality.

By contrast, convective weather systems and storms in particular can extract work only via convective-not conductive or radiative-neutralization of temperature gradients. Moreover by Sandström's theorem [59] (in Reference [59] see Section 1.5) convection is possible only if the heat source is at a higher pressure (and hence at a lower altitude) than the heat sink, implying that the spatial direction of the temperature gradient is material: it must be a vertical gradient with temperature decreasing upwards. Thus moist-atmosphere convective weather systems and storms such as cumuliform clouds, thunderstorms, tornadoes, and tropical cyclones can extract work only at the expense of the moist-convectively-available, i.e., super-moist-adiabatic, portion of vertical temperature gradients. Hence they can utilize only partial neutralization of vertical temperature gradients to moist-adiabaticity, not total neutralization to isothermality. However, this is more complete utilization of vertical temperature gradients than is possible in a dry atmosphere, wherein only the $d r y$-convectively-available, i.e., super- $d r y$-adiabatic, portion thereof can be utilized, i.e., partial neutralization only to dry-adiabaticity-the best that dry-atmosphere convective weather systems and storms such as dust devils [60-63] can do (see also Reference [41], p. 361; and Reference [42], p. 143). Dust devils can extract work only at the expense of the $d r y$-convectively-available, i.e., super-dry-adiabatic, portion of vertical temperature gradients and thus require vertical temperature gradient exceeding 
the dry adiabatic; cumuliform clouds, thunderstorms, tornadoes, and tropical cyclones merely exceeding the moist adiabatic. What is more, super-moist-adiabatic lapse rates are commonly sustained throughout a large fraction of the depth or even the entire depth of the troposphere, but super-dry-adiabatic lapse rates typically only through a shallow depth immediately above a hot (most usually desert) surface. Thus the moist-convectively-available free energy represented by a lapse rate in excess of the moist adiabatic exceeds by this ratio of depths the dry-convectively-available free energy represented by a lapse rate equally in excess of the dry adiabatic.

The convectively-available portion of lapse rates (the super-moist-adiabatic portion of lapse rates in a saturated atmosphere, and the super-dry-adiabatic portion thereof in a dry one) is sometimes referred to as representing hydrodynamic instability. The totality of lapse rates is sometimes referred to as representing thermodynamic instability. Heat engines in general can utilize the entirety of thermodynamic instability irrespective of Sandström's theorem [59]; convective weather systems and storms in particular only the hydrodynamic-instability portion thereof and even that only in accordance with Sandström's theorem [59].

We should note that even moist-atmosphere convective weather systems and storms such as cumuliform clouds, thunderstorms, tornadoes, and tropical cyclones require an at least marginally super-dry-adiabatic lapse rate to initiate convection from Earth's (land or ocean) surface to the lifting condensation level, if no other source of free energy is available to do so. If another source of free energy is available, e.g., turbulence caused by imported wind interacting with Earth's surface (recall the last paragraph of Section 2), then the required initial dry convection can be forced even in the face of a sub-dry-adiabatic lapse rate from Earth's surface to the lifting condensation level. Once initiated, all but the weakest moist-atmosphere convective weather systems and storms can via their own winds generate the required dry convection from Earth's surface to the lifting condensation level.

A brief aside concerning Sandström's theorem [59]: Sandström's theorem as usually stated, i.e., that convection is possible only if the heat source is at a higher pressure (and hence at a lower altitude) than the heat sink, is valid for any fluid whose density decreases with increasing temperature at constant pressure. This always obtains for gases and almost always for liquids. Fresh water (not sea water) between $0^{\circ} \mathrm{C}$ and $4{ }^{\circ} \mathrm{C}$ is a rare exception: within this limited temperature range its density increases with increasing temperature at constant $(1 \mathrm{~atm})$ pressure. However, Sandström's theorem encompasses such rare exceptions if it is stated somewhat more generally as follows: Convection is possible in a fluid whose density decreases (increases) with increasing temperature at constant pressure only if the heat source is at a higher (lower) pressure (and hence at a lower (higher) altitude) than the heat sink.

\section{Kinetic Enhancement in a Saturated Atmosphere}

Vertical temperature lapse rates can be utilized not only more completely thermodynamically in a saturated atmosphere than in a dry one, but also faster kinetically. The large heat of condensation (and to a lesser extent fusion) of water thermodynamically facilitates expenditure of vertical temperature gradients and hence of the free energy represented thereby to the moist adiabatic, as opposed to expenditure only to the dry adiabatic that is possible without it. In fact a larger portion of free energy that is itself typically larger by the ratio of depths as per the last two sentences of the second paragraph of Section 4. To boot, the large heat of condensation (and to a lesser extent fusion) of water also accelerates upward heat transfer in Earth's troposphere. Thus it also facilitates expenditure of vertical temperature gradients and hence of the free energy represented thereby faster kinetically than would be possible without it: Not only more but also faster heat transfer from Earth's surface to the tropopause is possible in a saturated atmosphere than from Earth's surface to altitudes typically far short of the tropopause in a dry one. This accelerated utilization of super-moist-adiabatic temperature gradients-accelerated cooling of Earth's (land or ocean) surface and accelerated warming of the tropopause-yields accelerated extraction of work. Accelerated work extraction is increased power. This utilization can be further accelerated-further increasing the work-extraction rate or power-via 
mechanisms that entail positive feedback such as CISK and WISHE in tropical cyclones and hot towers in equatorial thunderstorms. (Holton and Hakin briefly but concisely discuss WISHE and CISK on pp. 318-321 and hot towers on pp. 378-384 of Reference [45]. More detailed discussions are provided in references cited for Chapters 9 and 11 of Reference [45].) However, it should be emphasized that such positive-feedback mechanisms are consumers-not producers-of super-moist-adiabatic surface-tropopause temperature gradients and hence of the free energy embodied therein. They are in fact super-consumers: They facilitate expenditure of super-moist-adiabatic surface-tropopause temperature gradients and hence of the free energy embodied therein in a saturated atmosphere faster than would be possible without them in a saturated atmosphere. Thermodynamic ability to expend lapse rates down to the moist adiabatic as opposed to only down to the dry adiabatic and the greater depths through which super-moist-adiabatic lapse rates are typically maintained, as well as kinetically accelerated expenditure thereof via heat of condensation (and to a lesser extent fusion) facilitating faster upward heat transfer in Earth's troposphere, renders saturated-atmosphere convective weather systems and storms such as thunderstorms, tornadoes, and tropical cyclones more powerful than dry-atmosphere ones such as dust devils. Further kinetic acceleration of this expenditure via positive-feedback mechanisms such as CISK and WISHE in tropical cyclones and hot towers in equatorial thunderstorms renders them even more powerful yet. However, the greater the power, the faster the neutralization of the very temperature gradients-the faster the cooling of Earth's hot (land or ocean) surface and warming of the cold tropopause-that feeds convective weather systems and storms. Neither convective weather systems and storms nor these positive-feedback mechanisms can exist if the lapse rate is sub-moist-adiabatic-not even if the entire troposphere is saturated at $100 \%$ relative humidity and however high an absolute humidity, with however high an equivalent potential temperature $\theta_{e}$ (recall the third paragraph of Section 2).

Thermodynamic disequilibria (a) between the top layer of the tropical ocean and the lowest layer of the troposphere, and (b) owing to super-moist-adiabatic lapse rates within the troposphere itself, are required for WISHE and CISK to operate-indeed for tropical cyclones to operate with or without help from WISHE and/or CISK. Disequilibrium (a) can obtain via the top layer of the tropical ocean being warmer than lowest layer of the troposphere and/or the relative humidity in the lowest layer of the troposphere being less than $100 \%$ (to be exact, less than the very-slightly-less-than- $100 \%$ saturation value with respect to slightly-salty sea water (recall Item (c) of the first paragraph of Section 2)). Thus disequilibria (a) and (b) together comprise the overall required vertical thermodynamic disequilibrium. Its expenditure tends to cool Earth's (ocean) surface and warm the tropopause-in accordance with Nature's abhorrence of gradients. We note that Holton and Hakin consider WISHE to be more important than CISK in the development of tropical cyclones (see Reference [45], Section 9.7.2). However, neither WISHE nor CISK can operate, nor can hot towers exist, if the lapse rate is sub-moist-adiabatic-not even if the entire troposphere is saturated at $100 \%$ relative humidity and however high an absolute humidity, with however high an equivalent potential temperature $\theta_{e}$ (recall the third paragraph of Section 2).

Efficiency at maximum power output is less-but even at worst only moderately less—than the Carnot value $\epsilon_{\text {Carnot }}=1-\frac{T_{\text {cold }}}{T_{\text {hot }}}$. The Carnot value assumes reversible heat flows, i.e., infinitesimal temperature differences between hot reservoir and heat-engine input and between heat-engine exhaust and cold reservoir, and neglects friction. (Convective weather systems and storms are heat engines with heat input at Earth's surface and exhaust at higher tropospheric altitudes, up to the tropopause.) Assuming endoreversibilty (allowing finite temperature differences and hence irreversible heat flows between hot reservoir and heat-engine input and between heat-engine exhaust and cold reservoir, but neglecting friction), if heat flows are directly proportional to temperature differences the Curzon-Ahlborn efficiency $\epsilon_{\mathrm{CA}}=1-\left(\frac{T_{\text {cold }}}{T_{\text {hot }}}\right)^{1 / 2}$ [64-67] obtains at maximum power output. (In Reference [65] see Section 4-9. While Curzon and Ahlborn derived $\epsilon_{\mathrm{CA}}$ independently [64,65], it had been derived previously [66,67].) Although $\frac{\epsilon_{\mathrm{CA}}}{\epsilon_{\text {Carnot }}}=\left[1-\left(\frac{T_{\text {cold }}}{T_{\text {hot }}}\right)^{1 / 2}\right] \div\left(1-\frac{T_{\text {cold }}}{T_{\text {hot }}}\right)$ decreases 
monotonically with increasing $\frac{T_{\text {cold }}}{T_{\text {hot }}}$, it is never less than $\frac{1}{2}$. In the limit $\frac{T_{\text {cold }}}{T_{\text {hot }}} \rightarrow 0, \frac{\epsilon_{\mathrm{CA}}}{\epsilon_{\text {Carnot }}} \rightarrow 1$; in the limit $\frac{T_{\text {cold }}}{T_{\text {hot }}} \rightarrow 1, \frac{\epsilon_{\mathrm{CA}}}{\epsilon_{\text {Carnot }}} \rightarrow \frac{1}{2}$. (The latter limit is most easily verified by setting $\frac{T_{\text {cold }}}{T_{\text {hot }}}=1-\delta$; in the limit $\delta \rightarrow 0$ with the help of the binomial theorem $\left[1-(1-\delta)^{1 / 2}\right] \div[1-(1-\delta)]=\left[1-(1-\delta)^{1 / 2}\right] \div \delta \rightarrow$ $\left[1-\left(1-\frac{1}{2} \delta\right)\right] \div \delta=\frac{1}{2} \delta \div \delta=\frac{1}{2}$.) Efficiency at maximum power output (assuming heat flows directly proportional to temperature differences between hot reservoir and engine input and between engine exhaust and cold reservoir [64-67] or more generally [68-72]) has been studied both for macroscopic heat engines (which all atmospheric ones are, and hence which are of primary interest to us) [64-70], and microscopic ones [71,72]. Efficiency at maximum work output per cycle of macroscopic heat engines has also been studied [73].

\section{Synopsis}

It is often stated that primary energy source for just about everything meteorological is the Sun-heat from the Sun. However, of course it is understood that the Sun alone is not enough, and that heat alone is not enough. A smeared-out Sun [74] (in Reference [74] see "Smeared-Out Sun" on p. 249 and "If The Sun Were Smeared Out" on p. 250) would provide just as much heat but zero work and hence there would then be nothing meteorological [74]. Equally important to the non-smeared-out Sun [74] being hot and bright at $5800 \mathrm{~K}$ is the rest of the sky being cold and dark at the $2.7 \mathrm{~K}$ temperature of the cosmic background radiation [24]. The Sun heating the atmosphere primarily at Earth's surface as required by Sandström's theorem [59]—-because air is largely transparent and Earth's surface opaque to sunlight-is not enough. Equally important is the coldness of the $2.7 \mathrm{~K}$ cosmic-background-radiation heat sink that allows radiation to space and hence a cold tropopause to exist [24]. Otherwise temperature could not decrease with increasing altitude faster than the moist-adiabatic lapse rate-or indeed at all. The disequilibrium owing to vertical temperature gradients between Earth's surface and the tropopause exists only at the expense of the even greater disequilibrium between the $5800 \mathrm{~K}$ solar disk and the $2.7 \mathrm{~K}$ cosmic background radiation [24]. It is this $2.7 \mathrm{~K}$ low-energy-photon heat sink that allows Earth to easily radiate approximately 20 intermediate-energy $\approx 300 \mathrm{~K}$ photons into space for every high-energy $5800 \mathrm{~K}$ photon received from the Sun, with the consequent increase in entropy paying for virtually all organized (nongeothermally-driven and nontidal) activity-meteorological and otherwise-on Earth [24]. Meteorology is enabled by work, not by heat; hence by the disequilibrium between the $5800 \mathrm{~K}$ solar disk and the $2.7 \mathrm{~K}$ cosmic background radiation [24]—-by delocalization of energy from the solar disk to Earth and thence from Earth to space [24] — not by energy per se and not by the Sun alone.

Insolation heating Earth's surface in accordance with Sandström's theorem [59] and radiative cooling of the tropopause to space enables continuous regeneration of super-moist-adiabatic vertical temperature gradients. These gradients in turn enable all (moist-atmosphere) convective weather systems and storms. With or without positive-feedback mechanisms, condensation is never a thermodynamically spontaneous process in Earth's atmosphere. It always yields heat but always costs work and hence is never a driver but always a passenger. It is an important passenger-heat of condensation certainly is important in the thermodynamics of convective weather systems and storms, but nevertheless still a passenger that costs work to enable. The work that it costs to enable convection and hence the side result of supersaturation, and thence condensation and consequent release of heat of condensation, is paid for by the work yielded by the driver-by (partial) neutralization of super-moist-adiabatic temperature gradients to moist-adiabaticity tending to cool Earth's (land or ocean) surface and warm the tropopause-by Nature's abhorrence of gradients. (Recall Sections 2 and 4.) The large heat of condensation (and to a lesser extent fusion) of water facilitates expenditure of vertical temperature gradients not only more completely thermodynamically but also faster kinetically than would be possible without it. Positive-feedback mechanisms facilitate this expenditure even faster yet kinetically. (Recall Section 5.) However, with or without positive-feedback mechanisms, super-moist-adiabatic vertical temperature gradient is the driver that enables convection 
and thence in turn supersaturation, condensation, and consequent release of heat of condensation. Thus condensation and consequent release of heat of condensation is the passenger, albeit an important passenger. Convection and hence the side result of supersaturation, and thence condensation and consequent release of heat of condensation, cannot exist, and thus neither convective weather systems and storms nor positive-feedback mechanisms can exist, if the lapse rate is sub-moist-adiabatic-not even if the entire troposphere is saturated at $100 \%$ relative humidity and however high an absolute humidity, with however high an equivalent potential temperature $\theta_{e}$ (recall the third paragraph of Section 2).

We re-iterate (recall the third-to-last paragraph of Section 1) that the very properties of water help to maintain super-moist-adiabatic lapse rates in Earth's troposphere: Water vapor, because it is a greenhouse gas, raises temperatures at Earth's surface via reradiation of infrared frequencies from Earth's atmosphere (mainly from the troposphere) back to Earth's surface, and enhances radiation of infrared frequencies to space from tropospheric altitudes well above Earth's surface thus lowering temperatures at these higher altitudes. Condensed into clouds, water helps to maintain nighttime ambient super-moist-adiabatic lapse rates in the air surrounding the clouds by acting as a blanket trapping infrared radiation from Earth's surface and reradiating it back to Earth's surface, thus raising nighttime temperatures at Earth's surface, while infrared radiation to space from cloud tops, which are better radiators than air, helps to lower nighttime temperatures there. (Of course, during the daytime, clouds usually lower temperatures at Earth's surface: reduced insolation usually more than offsets the heat-blanket effect.)

In the long run Earth and its atmosphere are in steady-state; in the long run expenditure of vertical temperature gradients is matched by their regeneration, ultimately at the expense of the disequilibrium between the $5800 \mathrm{~K}$ solar disk and the $2.7 \mathrm{~K}$ cosmic background radiation [24]. However, in the short run, convective weather systems and storms can expend these gradients (at any rate the super-moist-adiabatic portion thereof in the moist-atmosphere case and the super-dry-adiabatic portion thereof in the dry-atmosphere case) faster than they can be regenerated, by expending capital or savings in addition to income.

Acknowledgments: I am grateful to Donald H. Kobe, Marlan O. Scully, and Paolo Grigolini for their encouragement to work on this topic and for their very helpful suggestions and comments. I am also grateful to all three reviewers of the initial version of my manuscript for Entropy. I thank the first reviewer of the initial version of my manuscript for a very helpful list of references, from among which are References $[2,24,42,43,75,76]$ cited in this revised version thereof. (Reference [76] by extension requires also citing Reference [77].) I also thank Technical Support at Mackichan Software, especially John MacKendrick and George Pearson, for their extensive help with putting the initial version of my manuscript into the correct computer format, into which I could paste early drafts of this revised version thereof. I am also thankful to all three reviewers of this revised version of my manuscript for Entropy and to Eliodoro Chiavazzo and Kevin H. Knuth, Academic Editors for Entropy, for their very constructive help, suggestions, and advice concerning it. I thank the first reviewer of this revised version of my manuscript for informing me of References [25,27,29,30]. (Reference [25] by extension requires also citing Reference [26]. These new references helped me to also find Reference [28].) I thank the second reviewer of this revised version of my manuscript for informing me of Reference [78]. (Reference [78] by extension requires also citing Reference [79], and helped me to also find Reference [80].) I thank the third reviewer of this revised version of my manuscript for emphasizing Reference [3], which led to citing both it and Reference [4]. I thank Technical Support at Mackichan Software, especially John MacKendrick and George Pearson, for their help with computer-related questions concerning this revised version of my manuscript. Additionally, I thank S. Mort Zimmerman for engaging in very interesting scientific discussions over many years, Dan Zimmerman and Kurt W. Hess for engaging in very interesting scientific discussions at times, and Robert $\mathrm{H}$. Shelton for very helpful advice concerning diction.

Conflicts of Interest: The author declares no conflict of interest.

\section{Appendix A. Horizontal Temperature Gradients}

In this paper our focus has been on vertical temperature gradients, and on weather systems that derive their free energy entirely or at least primarily at the expense of vertical temperature gradients, mainly moist-atmosphere ones such as cumuliform clouds, thunderstorms, tornadoes, and tropical cyclones, but for purposes of comparison also dry-atmosphere dust devils. However, a few brief remarks concerning horizontal temperature gradients are in order. The disequilibrium owing to 
horizontal temperature gradients, as owing to vertical ones, exists only at the expense of the even greater disequilibrium between the $5800 \mathrm{~K}$ solar disk and the $2.7 \mathrm{~K}$ cosmic background radiation [24]. Weather systems such as extratropical cyclones and anticyclones (see Reference [41], Sections 1.3.5 and 8.1; and Reference [45], Chapters 6, 7, and 10), and sea and land breezes (see Reference [41], Section 9.5.2; and Reference [45], p. 99), derive their free energy entirely or at least primarily at the expense of horizontal temperature gradients. It is sometimes stated that the primary meteorological energy source is horizontal latitudinal temperature gradient outside the tropics and latent heat of condensation in the tropics (see Reference [45], the second paragraph of Chapter 11 on p. 377). A more correct statement is that the primary meteorological free-energy source is horizontal latitudinal temperature gradient outside the tropics and vertical temperature gradient exceeding the moist adiabatic in the tropics. For it costs work-the work that it costs to enable convection-to force supersaturation and thence condensation and consequent yielding of latent heat of condensation: supersaturation and thence condensation and consequent yielding of heat of condensation is a thermodynamically nonspontaneous process that must be forced. It is forced at the expense of diminution of super-moist-adiabatic temperature gradient, at the expense of tending to cool Earth's (land or ocean) surface and warm the tropopause-in accordance with Nature's abhorrence of gradients. Holton and Hakin correct any misunderstanding that may result from the second paragraph of Chapter 11 on p. 377 of Reference [45] in Section 11.3 thereof, where they clearly state that ascent of saturated air yields work and hence is thermodynamically spontaneous if the lapse rate is super-moist-adiabatic, but costs work and hence is thermodynamically nonspontaneous and consequently must be forced if the lapse rate is sub-moist-adiabatic.

We should note that the horizontal temperature gradient in tropical cyclones, with temperature increasing inwards towards the eye, has been construed as their immediate free-energy source (see Reference [81], pp. 82-85, pp. 90-92, Chapter 6 (especially pp. 123-127), pp. 202-204, and pp. 285-291). However, it is recognized (see Reference [39], the Appendix; and Reference [81], pp. 126-127, 129-134, and 202-204) that this horizontal temperature gradient exists only at the expense of release of heat of condensation being concentrated near the eye, which in turn occurs only at the expense of a super-moist-adiabatic vertical temperature environment that enables convection and thus in turn supersaturation, condensation, and release of heat of condensation. This super-moist-adiabatic vertical temperature environment is the next-to-ultimate free energy source (the ultimate one is of course the $5800 \mathrm{~K}$-solar-disk/2.7 K-cosmic-background-radiation disequilibrium [24]). Forced dry-adiabatic descent of air in the eye further raises the temperature in the eye but costs free energy. It occurs only because ascent of saturated air at the expense of a super-moist-adiabatic environment outside the eye yields more free energy. Thus the secondary, smaller, horizontal temperature difference between the warm eye and cooler periphery of a tropical cyclone exists only at the expense of the much larger, primary, super-moist-adiabatic vertical temperature difference between Earth's warm (ocean) surface and the cold tropopause, which enables convection, and thus in turn supersaturation, condensation, and release of heat of condensation, within tropical cyclones.

Horizontal and vertical temperature gradients need not be independent. For example, horizontal temperature gradients facilitate sufficient heat flux into Antarctica to keep surface winter temperatures there well above that of the $2.7 \mathrm{~K}$ cosmic background radiation [24]. This contributes, along with radiational cooling from high atmospheric altitudes to space, to lapse rates above the surface inversion layer in Antarctica in winter that can reach the dry adiabatic [78,79], although on the average they are perhaps about half that large [80]. (In Reference [80] see Section 1.1 (The Thermal Structure of the Atmosphere), especially Figure 1.1 and the associated discussions.)

\section{Appendix B. Why Fractal Structure of Vertical Temperature Lapse Rates May Not Matter (at Least Not Much)}

It has been pointed out that the atmosphere, being a highly complex system far from thermodynamic equilibrium, may have a fractal structure that repeats on ever-smaller scales (scale 
invariance) [75-77]. (See also Reference [24], Chapters 2-5, especially Chapter 4, and most especially pp. 54-56.) For example, with respect to vertical temperature lapse rates, this implies that variations in lapse rate may repeat on ever-smaller scales: A stable macroscopic atmospheric layer could incorporate much thinner unstable sublayers, with each unstable sublayer incorporating much-thinner-yet stable sub-sublayers, etc. Likewise, an unstable macroscopic atmospheric layer could incorporate much thinner stable sublayers, with each stable sublayer incorporating much-thinner-yet unstable sub-sublayers, etc.

Of course, such a fractal structure cannot continue over an infinite range of scales: the vertical extent of the troposphere sets an upper limit, and the average distance between atmospheric (specifically, tropospheric) gas molecules a lower one. However, it can continue over a moderate number of decades of scale. The upper limit on this moderate number certainly can never be more than about a dozen, and is probably always somewhat smaller.

Thus to this extent, since in this paper we always assume smoothed-out lapse rates over macroscopic vertical distances, our discussions concerning lapse rates are only approximations. However, for the following two reasons, hopefully smoothed-out lapse rates over macroscopic vertical distances are a sufficiently good approximation for our purposes: (i) Free energy is a monotonically increasing function of lapse rate. (ii) Convection currents usually have enough inertia to easily punch through very thin stable sublayers, even more easily through even-thinner stable sub-sublayers, even more easily yet through even-thinner-yet stable sub-sub-sublayers, etc., that may exist within a fractal structure of a saturated macroscopic layer of the troposphere whose smoothed-out lapse rate is unstable super-moist-adiabatic, or of an unsaturated one whose smoothed-out lapse rate is unstable super-dry-adiabatic. Conversely, convection currents in very thin unstable sublayers usually do not have enough inertia to punch through embedding stable layers; much less so convection currents in even-thinner unstable sub-sublayers, much less so yet those in even-thinner-yet unstable sub-sub-sublayers, etc.

\section{Appendix C. Interpretations of Entropy}

There are, of course, many interpretations of entropy, and also criticisms of these interpretations (see for example Reference [14], Chapter 1, especially Sections 1.2-1.5). (But the interpretation of entropy as disorder (even given that disorder is a rather vague notion) in the case of entropy increasing via spontaneous crystallization from a supersaturated solution accompanied by a decrease in temperature can be defended by noting that correlations between solvent and solute molecules must then be weakened (see Reference [40], pp. 150-152).)

In accordance with the third law of thermodynamics, the zero of entropy obtains at $0 \mathrm{~K}$ given a nondegenerate ground state. Ultimately it may be true that the ground state of any system is nondegenerate [82]. (In Reference [82] see Postulate IV and the associated discussions on p. 27 and Chapter 10, especially Sections 10.1 and 10.4. Also see, in Reference [65], Postulate IV and the associated discussions on p. 30 and Chapter 11, especially Section 11-3. Postulate IV is restated on p. 284 of Reference [65] and on p. 192 of Reference [82]. (The cross-reference to Chapter 10 in the second-to-last line of the second-to-last paragraph on p. 30 of Reference [65] is a typo; it should be to Chapter 11.)) There is of course no thermal agitation at $0 \mathrm{~K}$; nonetheless a material system trapped in a metastable above-ground state at $0 \mathrm{~K}$ will quantum-mechanically tunnel to its ground state in finite time. (Of course, the energy thereby released cannot be dissipated as heat if the temperature is to remain $0 \mathrm{~K}$, and must be expelled from the system if it is to remain in its ground state.) In any case, certainly a vacuum is in a nondegenerate ground state at $0 \mathrm{~K}$.

However, in meteorology it is often more convenient to normalize entropies to the $1000 \mathrm{mbar}$ pressure surface. The potential temperature $\theta$ in an unsaturated atmosphere, and the equivalent potential temperature $\theta_{e}$ in a saturated one, is sometimes referred to as the meteorologist's entropy (see for example Reference [40], Sections 4.3 and 6.5-6.6). The potential temperature $\theta$ (the temperature of air if brought dry-adiabatically to a pressure of $1000 \mathrm{mbar}$ - and hence the entropy—is conserved in 
reversible dry-adiabatic ascents and descents. The actual temperature in such ascents and descents obeys the dry-adiabatic lapse rate. The equivalent-potential temperature $\theta_{e}$ (the temperature of air if brought moist-adiabatically to a pressure of $1000 \mathrm{mbar}$ - - and hence the entropy-is conserved in reversible moist-adiabatic ascents and descents if all condensed water is retained within ascending air parcels. The actual temperature in such ascents and descents obeys the moist-adiabatic lapse rate (sometimes referred to as the saturated-adiabatic lapse rate). The slightly larger psuedo-moist-adiabatic lapse rate corresponds to all condensed water precipitating out (see for example Reference [40], Section 6.5). For simplicity in this paper we have disregarded the small difference between these two lapse rates and referred simply to the moist-adiabatic lapse rate.

The meteorologist's entropy, summarized in the immediately preceding paragraph, is very useful for meteorological calculations. However, it seems not to be the interpretation of entropy that best conveys its physical meaning. The interpretation that best invests the concept of entropy with physical meaning, at least for our purposes, seems to be that most strongly emphasized by Reiss in Reference [3], Sections I.8 and IV.1-IV.6. It is more conveniently stated in terms of negentropy rather than entropy, and is as follows: Negentropy is the degree of constraint. Thus entropy is to be interpreted as the degree of lack of constraint, but this seems more awkward: as per Reference [3], p. 57 "certainly it is easier to think of the degree of constraint rather than the degree of lack of constraint". Negentropy can equivalently be interpreted as the degree of localization: to constrain is to localize. Localization can be in position space, in momentum space, or both; or to within a subset of an ultimately accessible set of quantum states or microstates. (Thus, for example, interaction, by correlating a system with its surroundings, constrains or localizes the (system + surroundings) into fewer quantum states or microstates; hence (recall the paragraph containing Equation (3) and the last paragraph of Section 3) $-S_{\text {interaction }}$ is a negative contribution to $S_{\text {total }}$ (which is negligible in all cases of interest to us, but which nevertheless exists).) Constraint, or equivalently localization, seems to be a much sharper and much more easily quantifiable concept than the rather vague notion of disorder, which is often used as a descriptor of entropy. Thus the second law of thermodynamics can be stated as follows: Net imposition of a constraint is impossible. A constraint can be imposed but only at the expense of greater (in the (unattainable) limit of perfection or reversibility, equal) relaxation of another constraint. Or, equivalently: Net localization is impossible. Localization can be imposed but only at the expense of greater (in the (unattainable) limit of perfection or reversibility, equal) delocalization elsewhere in phase space. This is the interpretation of entropy, or, equivalently, negentropy, that we have taken in this paper (recall especially the last paragraph of Section 3). (In this regard, remarking on pp. 150-152 of Reference [40] and the second sentence of this Appendix C, lowering the temperature decreases entropy not because the peak of the Maxwellian speed distribution moves to a lower value, but because molecular thermal speeds become more localized about the peak, i.e., because the width at half maximum is decreased.)

This is not to say that in some cases other interpretations of entropy may be preferable (we mentioned the meteorologist's entropy) or even provide a more helpful physical interpretation thereof. However, for our purposes the interpretation of entropy given by Reiss seems to be the best physical interpretation thereof.

But we re-iterate that thermodynamics can be based on the concept of work [8-11], rather than on the concept of entropy. The advantage of this approach is that work is the most basic, simplest, most sharply defined, and most familiar concept in thermodynamics [8-11], and all more complex thermodynamic concepts, such as energy, heat, entropy, and temperature can be derived from the concept of work [8-11]. 


\section{References}

1. Guggenheim, E.A. Thermodynamics: An Advanced Treatment for Chemists and Physicists, 7th ed.; North-Holland: Amsterdam, The Netherlands, 1985; Chapter 1.

2. Berry, R.S.; Rice, S.A.; Ross, J. Physical Chemistry, 2nd ed.; Oxford University Press: Oxford, UK, 2000; Chapters 12-19.

3. Reiss, H. Methods of Thermodynamics; Blaisdell: New York, NY, USA, 1965.

4. Pippard, A.B. The Elements of Classical Thermodynamics; Cambridge University Press: Cambridge, UK, 1981.

5. Maham, B.M.; Myers, R.J. University Chemistry, 4th ed.; Benjamin-Cummings: Menlo Park, CA, USA, 1987, Chapter 8.

6. Baierlein, R. Thermal Physics; Cambridge University Press: Cambridge, UK, 1999.

7. Schroeder, D.V. Thermal Physics; Addison Wesley Longman: San Francisco, CA, USA, 2000.

8. Atkins, P.; de Paula, J. Physical Chemistry, 7th ed.; Freeman: New York, NY, USA, 2002; Chapters 2-5.

9. Atkins, P.; de Paula, J. Physical Chemistry, 10th ed.; Oxford: New York, NY, USA, 2014.

10. Allahverdyan, A.E.; Nieuwenhuizen, T.M. A mathematical theorem as the basis for the Second Law: Thomson's formulation applied to equilibrium. Physica A 2002, 305, 542-552.

11. Mallinckrodt, A.J.; Leff, H.S. All about work. Am. J. Phys. 1992, 60, 356-364.

12. Sheehan, D.P. (Ed.) Quantum Limits to the Second Law; American Institute of Physics: Melville, NY, USA, 2002; Volume 643.

13. Nikulov, A.V.; Sheehan, D.P. (Eds.) Special Issue on Quantum Limits to the Second Law of Thermodynamics. Entropy. 2004, 6 (1).

14. Č́pek, V.; Sheehan, D.P. Challenges to the Second Law of Thermodynamics: Theory and Experiment; Springer: Berlin/Heidelberg, Germany, 2005.

15. Sheehan, D.P. (Ed.) The Second Law of Thermodynamics: Foundations and Status. Found. Phys. 2007, 37 (12).

16. Sheehan, D.P. (Ed.) Second Law of Thermodynamics: Status and Challenges; American Institute of Physics: Melville, NY, USA, 2011; Volume 1411.

17. Sheehan, D.P. (Ed.) Forthcoming Special Issue of Entropy: Limits to the Second Law of Thermodynamics: Experiment and Theory.

18. Tolman, R.C. Relativity, Thermodynamics, and Cosmology; Oxford University Press: Oxford, UK, 1934.

19. Resnick, R.; Halliday, D.; Krane, K.S. Physics, 10th ed.; John Wiley \& Sons: Hoboken, NJ, USA, 2014.

20. Friction. Available online: https://en.wikipedia.org/wiki/Friction (accessed on 18 November 2016).

21. Friction and Friction Coefficients. Available online: http://www.engineeringtoolbox.com/frictioncoefficients \-d_778.html (accessed on 18 November 2016).

22. Rolling resistance. Available online: https://en.wikipedia.org/wiki/Rolling_resistance (accessed on 18 November 2016).

23. Rolling Resistance. Available online: www.engineeringtoolbox.com/rolling-friction-resistance-d_1303.html (accessed on 18 November 2016).

24. Tuck, A.F. Atmospheric Turbulence: A Molecular Dynamics Perspective; Oxford University Press: Oxford, UK, 2008; Section 3.4.

25. Pauluis, O.; Held, I.M. Energy Budget of an Atmosphere in Radiative-Convective Equilibrium. Part I: Maximum Work and Frictional Dissipation. J. Atmos. Sci. 2002, 59, 129-139

26. Pauluis, O.; Held, I.M. Energy Budget of an Atmosphere in Radiative-Convective Equilibrium. Part II: Latent Heat Transport and Moist Processes. J. Atmos. Sci. 2002, 59, 140-149.

27. Pauluis, O. Water Vapor and Mechanical Work: Comparison of Carnot and Steam Cycles. J. Atmos. Sci. 2011, $68,91-102$.

28. Goody, R. On the Mechanical Efficiency of Deep, Tropical Convection. J. Atmos. Sci. 2003, 60, $2827-2832$.

29. Laliberté, F.; Zika, J.; Mudryk, L.; Kushner, P.J.; Kejellsson, J.; Döös, K. Constrained work output of the moist atmospheric heat engine in a warming climate. Science 2015, 347, 540-543.

30. Pauluis, O. The Mean Air Flow as Lagrangian Dynamics Approximation and its Application to Moist Convection. J. Atmos. Sci. 2015, 73, 1-48.

31. Emanuel, K. Divine Wind; Oxford University Press: Oxford, UK, 2005.

32. Emanuel, K. Hurricanes: Tempests in a greenhouse. Physics Today 2006, 59, 74-75.

33. Emanuel, K. Tropical Cyclones. Annu. Rev. Earth Planet. Sci. 2003, 31, 75-104. 
34. Emanuel, K.A. Thermodynamic Control of Hurricane Intensity. Nature 1999, 401, 665-669.

35. Bister, M.; Emanuel, K.A. Dissipative Heating and Hurricane Intensity. Meteorol. Atmos. Phys. 1998, 65, $233-240$.

36. Emanuel, K. Response of tropical cyclone activity to climate change: Theoretical basis. In Hurricanes and Typhoons: Past, Present, and Future; Murnane, R.J., Liu, K.-B., Eds.; Columbia University Press: New York, NY, USA, 2004, pp. 395-407.

37. Emanuel, K.A.; Speer, K.; Rotunno, R.; Srivastava, R.; Molina, M. Hypercanes: A possible link in global extinction scenarios. J. Geophys. Res. 1995, 100, 13755-13765.

38. Emanuel, K.; Callaghan, J.; Otto, P. A hypothesis for the redevelopment of warm-core cyclones over Northern Australia. Mon. Weather Rev. 2008, 136, 3863-3872.

39. Denur, J. The apparent "super-Carnot" efficiency of hurricanes: Nature's steam engine versus the steam locomotive. Am. J. Phys. 2011, 79, 631-643.

40. Bohren, C.F.; Albrecht, B.A. Atmospheric Thermodynamics; Oxford University Press: Oxford, UK, 1998; Section 5.6.

41. Wallace, J.M.; Hobbs, P.V. Atmospheric Science, 2nd ed.; Academic Press: Amsterdam, The Netherlands, 2006; Sections 6.1 and 6.5 .

42. Ludlum, F.H. Clouds and Storms; The Pennsylvania State University Press: University Park, PA, USA; Chapter 5.

43. Richard, E.C.; Tuck, A.F.; Aiken, K.C.; Kelly, K.K.; Herman, R.L.; Troy, R.F.; Hovde, S.J.; Rosenlof, K.H.; Thompson, T.L.; Ray, E.A. High-resolution airborne profiles of $\mathrm{CH}_{4}, \mathrm{O}_{3}$, and water vapor near tropical Central America in late January to early February 2004. J. Goephys. Res. 2006, 111, D13304.

44. Thompson, P.D.; O’Brien, R. Weather; Time-Life Books: Alexandria, VA, USA, 1980; pp. 83-84.

45. Holton, J.R.; Hakin, G.J. An Introduction to Dynamic Meteorology, 5th ed.; Academic Press: Amsterdam, The Netherlands, 2013.

46. Reif, F. Fundamentals of Statistical and Thermal Physics; McGraw-Hill: New York, NY, USA, 1965.

47. Apertet, Y.; Ouerdane, H.; Goupil, C.; Lecoeur, P. Efficiency at maximum power of thermally coupled heat engines. Phys. Rev. E 2012, 85, 041144.

48. Bachhuber, C. Energy from the evaporation of water. Am. J. Phys. 1983, 51, 259-264.

49. Güémez, J.; Valiente, B.; Fiolhais, C.; Fiolhais, M. Experiments with the drinking bird. Am. J. Phys. 2003, 71, 1257-1264.

50. Abraham, N.; Paliffy-Muhoray, P. A dunking bird of the second kind. Am. J. Phys. 2004, 72, 782-785.

51. Lorenz, R. Finite-time thermodynamics of an instrumented drinking bird toy. Am. J. Phys. 2006, 74, 677-682.

52. Murrow, R.B. A Simple Heat Engine of Possible Utility in Primitive Environments. Available online: https: //www.rand.org/content/dam/rand/pubs/papers/2008/P3367.pdf (accessed on 18 November 2016).

53. Lear, J. New Waterbird for Egypt: A Robot Shadoof. Saturday Rev. 1967, 34, 49-50.

54. Murrow, R.B. The Research Frontier. Saturday Rev. 1967, 34, 51-55.

55. Craig, R.J. Modeling of a Thermodynamically Driven Heat Engine with Application Intended for Water Pumping. Master's Thesis, Stellenbosch University, Stellenbosch, South Africa, December 2014.

56. Chen, X.; Goodnight, D.; Gao, Z.; Cavusoglu, A.H.; Sabharwal, N.; DeLay, M.; Driks, A.; Sahin, O. Scaling up nanoscale water-driven energy conversion into evaporation-driven engines and generators. Nat. Commun. 2015, 6, 7346 .

57. Temming, M. Water, Water Everywhere. Sci. Am. 2015, 313, 26.

58. Ornes, S. Spore Power. In Discover 2016, 37, 14. Available online: www.DiscoverMagazine.com/HYDRA (accessed on 18 November 2016).

59. Houghton, J. The Physics of Atmospheres, 3rd ed.; Cambridge University Press: Cambridge, UK, 2002; Section 1.5

60. Ives, R.L. Behaviour of dust devils. Bull. Am. Meteorol. Soc. 1947, 28, 168-174.

61. Williams, N.R. Development of dust-whirls and similar small-scale vortices. Bull. Am. Meteorol. Soc. 1948, 29, 105-117.

62. Sinclair, P.C. Some preliminary dust-devil measurements. Mon. Weather Rev. 1964, 92, 363-367.

63. Malkus, J.S. Interchange of properties between sea and air: Large-scale interactions. In The Sea; Interscience: London, UK, 1962; Volume 1, p. 205.

64. Curzon, F.L.; Ahlborn, B. Efficiency of a Carnot engine at maximum power output. Am. J. Phys. 1975, $43,22-24$.

65. Callen, H.C. Thermodynamics and an Introduction to Thermostatistics; John Wiley \& Sons: Hoboken, NY, USA, 1985. 
66. Vaudrey, A.; Lanzetta, F.; Feidt, M.H.B. Reitlinger and the origins of the efficiency at maximum power formula for heat engines. J. Non-Equilib. Thermodyn. 2014, 39, 199-203.

67. Endoreversible Thermodynamics. Available online: https://en.wikipedia.org/wiki/Endoreversible_ thermodynamics (accessed on 18 November 2016).

68. De Vos, A. Efficiency of some engines at maximum-power conditions. Am. J. Phys. 1985, 53, 570-573.

69. Gordon, J.M. Maximum power point characteristics of heat engines as a general thermodynamic problem. Am. J. Phys. 1989, 57, 1136-1142.

70. Gordon, J.M. Observations on efficiency of heat engines operating at maximum power. Am. J. Phys. 1990, 58, 370-375.

71. Schmiedl T.; Seifert, U. Efficiency at maximum power: An analytically solvable model for stochastic heat engines. Eurphys. Lett. 2008, 81, 20003.

72. Tu, Z.C. Efficiency at maximum power of Feynman's ratchet as an engine. J. Phys. A 2008, 41, 312003.

73. Leff, H.F. Thermal efficiency at maximum work output: New results for old heat engines. Am. J. Phys. 1987, 55, 602-610.

74. Epstein, L.C. Thinking Physics: Understanding Practical Reality, 3rd ed.; Insight Press: San Francisco, CA, USA, 1999.

75. Lovejoy, S.; Tuck, A.F.; Hovde, S.J.; Schertzer, D. Do Stable Atmospheric Layers Exist? Geophys. Res. Lett. 2008, 35, L032122.

76. Tuck, A.F. Review Article: From Molecules to Meteorology Via Turbulent Scale Invariance. Q. J. R. Meteorol. Soc. 2010, 136, 1125-1144.

77. Tuck, A.F. Correction to: 'From Molecules to Meteorology Via Turbulent Scale Invariance'. Q. J. R. Meteorol. Soc. 2011, 137, 275.

78. Tuck, A.F. Synoptic and Chemical Evolution of the Antarctic Vortex in Late Winter and Early Spring, 1987. J. Geophys. Res. 1989, 94, 11687-11737.

79. Tuck, A.F. Correction to "Synoptic and Chemical Evolution of the Antarctic Vortex in Late Winter and Early Spring, 1987" by A. F. Tuck. J. Geophys. Res. 1989, 94, 16855-16856.

80. Ramaswamy, V.; Hurrell, J.W.; Meehl, G.A.; Phillips, A.; Santer, B.D.; Schwarzkopf, M.D.; Seidel, D.J.; Sherwood, S.C.; Thorne, P.W. Temperature Trends in the Lower Atmosphere. Available online: www.gfdl. noaa.gov/bibliography/related_files/vr0603.pdf (accessed on 18 November 2016).

81. Dunn, G.; Miller, B.I. Atlantic Hurricanes; Louisiana State University Press: Baton Rouge, LA, USA, 1964.

82. Callen, H.B. Thermodynamics; John Wiley \& Sons: Hoboken, NY, USA, 1960.

(C) 2016 by the author; licensee MDPI, Basel, Switzerland. This article is an open access article distributed under the terms and conditions of the Creative Commons Attribution (CC-BY) license (http:/ / creativecommons.org/licenses/by/4.0/). 